\title{
THE STRUCTURE OF THREATENED VEGETATION IN THE MONTANE TEMPERATE ECOSYSTEM OF PASHAT VALLEY, PAK-AFGHAN BORDER, HINDUKUSH RANGE, BAJAUR, PAKISTAN
}

\author{
HAQ, A. ${ }^{1,2^{*}}-$ BADSHAH, L. ${ }^{1}$

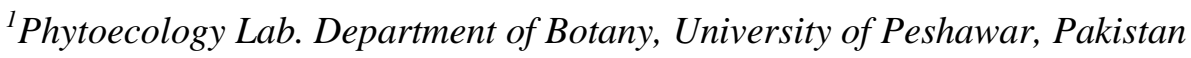 \\ ${ }^{2}$ Department of Botany, Govt. Post Graduate College Khar, District Bajaur, Pakistan \\ (phone: +92-346-318-2772 (Haq, A.); +92-333-894-4128 (Badshah, L.)) \\ ${ }^{*}$ Corresponding author \\ e-mail:aminulhaq_bot@yahoo.com \\ (Received $14^{\text {th }}$ Mar 2021; accepted $19^{\text {th }}$ Jul 2021)
}

\begin{abstract}
The present phytosociological study was conducted during 2017-2020 in Pashat valley, Pak-Afghan border, Bajaur, Pakistan. Fifteen communities were established, 5 for each herb, shrub and tree through the systematic random quadrat method, i.e., $10 \mathrm{~m}^{2}$ for trees, $5 \mathrm{~m}^{2}$ for shrubs and $1 \mathrm{~m}^{2}$ for herbs in the five selected sites. Three communities were documented in the respective sites for trees, shrubs and herbs viz Pinus-Quercus-Juglans, Berberis-Indigofera-Buddleja, Rumex-Hyparrhenia-Cynodon on site I, Pinus-Olea-Quercus, Berberis-Otostegia-Sarcococca and Hyparrhenia-Cynodon-Heteropogon on site II, Olea-Quercus, Rubus-Otostegia-Berberis and Cynodon-Salvia-Cyperus on site III, Quercus-Olea-Juglans, Sarcococca-Justicia-Otostegia and Cynodon-Conyza-Thymus on site IV and Quercu-Olea-Ficus, Otostegia-Berberis-Sageretia and Apluda-Cynodon-Chrysopogon on site V. Cluster analysis through PAST and PC-ORD classified it into four groups. Soil samples of the selected sites were physicochemically analyzed. The soil texture ranged from silty to clay loam with basic $\mathrm{pH}$ ranging from 7.3-7.8 and low organic matter. Nitrogen ( 0.036 to $0.044 \mathrm{mg} / \mathrm{kg}$ ), phosphorus $(7.40$ to $15.4 \mathrm{mg} / \mathrm{kg}$ ) and potassium (80 to $120 \mathrm{mg} / \mathrm{kg}$ ) were among the macronutrients of the soil. Deforestation and soil erosion in the area were the major threats to the phytodiversity.
\end{abstract}

Keywords: phytosociology, similarity index, maturity index, species diversity, montane temperate vegetation

\section{Introduction}

Phytosociology is the study of plant assemblages in communities (Dengler, 2016) and it is the best way to learn about structure, habit, niche and habitat of vegetation as well as plant interactions in an ecosystem (Khan et al., 2016). The establishment of plant communities and its composition depend on time and altitude within a region, however, latitude, slope, and precipitation are also key factors (Kharkwal et al., 2005). Phytosociological research is critical for understanding the plant population dynamics and their interactions with biotic and abiotic influences which is the foundation for conservation initiatives, regeneration of degraded areas and long-term forest resource management (Zerwes et al., 2018).

In ecological research, the relationship between abiotic and biotic components of an ecosystem is becoming extremely prevalent (Tavili and Jafari, 2009; Khan et al., 2016). Some species change their growth form, life cycle and development to adopt the changing conditions, resulting in changes in plant populations and thus facilitating the spread of invasive species (Rahman et al., 2016). A quantitative vegetation analysis in a forest ecosystem provides data on species diversity, community organization, niche resources 
distribution and species over rate (Mandal and Joshi, 2014). Plants that grow in close proximity have a mutual association with one another and with the surrounding environment (Mishra et al., 1997; Mandal and Joshi, 2014). The quantitative relationship between abundant and rarely growing plant species is an important property of a community. Quantitative analysis of vegetation aims to characterize the vegetation, predict its pattern and classify it in a meaningful way (Braun-Blanquet, 1932; Odum, 1971).

A complex set of both abiotic and biotic factors such as elevation, slope, participation, temperature, deforestation, erosion, trampling overgrazing and developmental works influence on the structure of vegetation of an area (Khan et al., 2011, 2017; Habib et al., 2014; Evangelista et al., 2016; Ali et al., 2018; Mota et al., 2018; Hussain et al., 2019; Bhat et al., 2020; Hailemariam and Temam, 2020; Zaman and Badshah, 2020; Yang et al., 2020). The first and the most important step of any ecological study of an area is to conduct a phytosociological evaluation of the plant population which is essential to understand the function of a community (Warger and Morrel, 1978). A great deal of phytosociological research has been documented in Pakistan. Khan et al. (2016) studied the phytosociology of Indus-Kohistan Pine forests. Haq et al. (2015) documented the vegetation of subtropical forests of district Bataghram. Ali et al. (2015) investigated the vegetation structure in Buner Khyber Pakhtunkhwa in relation to edaphic variables. Ali et al. (2018) studied the vegetation structure and threats to mountain ecosystem of Hindukush rang, Swat Pakistan. In the Koh-e-Safaid range, Kurram, Pakistan, Hussain et al. (2019) summarized seven communities of herbs, shrubs, and trees vegetation. The vegetation dynamics along the altitudinal gradient of Terich valley, Hindukush range, Chitral, Pakistan were studied by Zaman and Badshah (2020). Pashat valley lies in the famous Hindukush ranges of Pakistan having rich phytodiversity which is under high anthropogenic disturbance. The literature review clearly shows that there is no such work done on the Pashat valley which will fill the research gap in the area. There is an utmost need to study the area in phytosociological context which will be helpful for the conservation and sustainable utilization of plant resources of the area and also for future ecological study.

Therefore, the aim of this study was to analyse the structure of plant community in the Pashat valley Bajaur, and to identify the edaphic variables responsible for the establishment of plant communities and their distribution pattern.

\section{Materials and Methods}

\section{Study area}

Bajaur, a part of the former administrative setup of FATA (Federally Administrated Tribal Area), has been recently merged as a district in the Khyber Pakhtunkhwa province of Pakistan. It lies in the north of the country and nearly one half of its area has a hilly terrain. Some lofty mountains of the Hindu Kush range lie here, in the north and northwest, it shares a $52 \mathrm{~km}$ long border with Kunar Province of Afghanistan. The Pashat valley is located in Tehsil Salarzai at $34.45^{\circ} \mathrm{N}$ to $34.57^{\circ} \mathrm{N}$ latitude and $71.25^{\circ} \mathrm{E}$ to $71.35^{\circ}$ longitude. The valley has a high peak "the Latai Sar" which is the famous pass for transborder trade and transportation with Afghanistan. The area has plenty of water resources in spring and stream forms of which the Gaber Chena is the popular picnic spot in summer. The area is also famous for the cultivation of a local variety of rice (Oryza sativa) "the Begamai". Its altitude varies from $1300 \mathrm{~m}$ to more than $2600 \mathrm{~m}$ from sea level (Fig. 
1). The weather is moderate in summer but extremely cold in winter due to snowfall. The coldest months are December and January with mean temperature from $5{ }^{\circ} \mathrm{C}$ to $10^{\circ} \mathrm{C}$. The hottest month is July with mean temperature which varies $26^{\circ} \mathrm{C}$ to $40{ }^{\circ} \mathrm{C}$. The annual rainfall is approximately $500 \mathrm{~mm}$, with $375 \mathrm{~mm}$ falling in July and August.

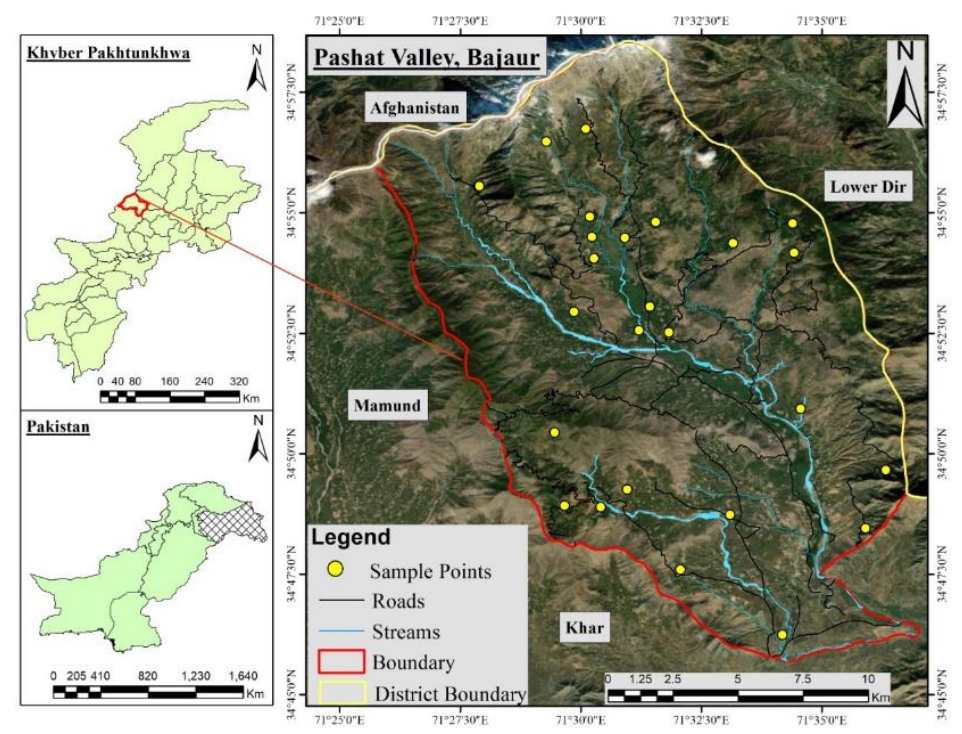

Figure 1. Map of the study area

\section{Quantitative analysis of communities}

Phytosociological studies were conducted at five different monitoring sites during 2017-2020. At each monitoring site, vegetation was examined using $5(10 \times 10 \mathrm{~m})$ quadrats for trees, $10(5 \times 5 \mathrm{~m})$ quadrats for shrubs, and $15(1 \times 1 \mathrm{~m})$ quadrats for herbs. The density, cover and frequency of each species were determined using formulae, then converted to relative values to get IV (Importance value) and FIV (Family importance value).

Density: The species density and its relative value were calculated using standard formulae (Oosting, 1956; Hussain, 1989).

$$
\begin{gathered}
\text { Density (D) }=\frac{\text { Total number of individuals of a species }}{\text { Total number of quadrates }} \\
\text { Relative Density (RD) }=\frac{\text { Number of individuals of a species }}{\text { Total density of all species }} \times 100
\end{gathered}
$$

Cover: The cover and relative cover of species were determined with specified formulae (Cox, 1967; Hussain, 1989).

$$
\begin{aligned}
\text { Cover }(C) & =\frac{\text { Sum of mid points of a species }}{\text { Total area sampled }} \\
\text { Relative cover }(\mathrm{RC}) & =\frac{\text { Cover of a species }}{\text { Total cover of all species in a stand }} \times 100
\end{aligned}
$$


Basal Area: Measuring tape is used for diameter of crown and recorded the value in square feet. The basal area was measured through using table of basal area (Cox, 1967; Hussain, 1989).

$$
\begin{gathered}
\text { Basal Area }(B A)=\frac{\text { Area of species calculated from circumference at DBH }}{\text { Total sampled area }} \\
\text { Relative Basal Area }(\text { RBA })=\frac{\text { Basal area of a given species }}{\text { Total area of all species in a stand }} \times 100
\end{gathered}
$$

Frequency: The frequency and relative frequency of the plants were determined by using the formulae given (Hussain, 1989).

$$
\begin{gathered}
\text { Frequency }(F)=\frac{\text { Number of quadrates in which a species present }}{\text { Total number of quadrates taken }} \times 100 \\
\text { Relative Frequency }(\mathrm{RF})=\frac{\text { Frequency of a species }}{\text { Total frequency of all species }} \times 100
\end{gathered}
$$

Importance value: The relative values of three parameters, namely density, cover and frequency, were added to determine the importance value of a species (Cox, 1967; Hussain, 1989).

$$
\mathrm{IV}=\mathrm{RD}+\mathrm{RC}+\mathrm{RF}
$$

Family Importance Value: FIV for all recorded families were calculated by adding the importance value of each species from each family.

Index of Similarity (IS): The similarity index was calculated by using Sorensen's index (1948), as revised by Motyka et al. (1950).

$$
\mathrm{IS}=\frac{2 \mathrm{~W}}{\mathrm{~A}+\mathrm{B}} \times 100
$$

where, 'IS' stands for index of similarity, 'W' stands for the lowest value that both A and $\mathrm{B}$ community have in common, ' $\mathrm{A}$ ' and ' $\mathrm{B}$ ' stands for species values in community $\mathrm{A}$ and $\mathrm{B}$, respectively.

Species Diversity ( $S D)$ : Simpson-Wiener index of similarity was used to quantify the species diversity in the stands (Simpson, 1961).

$$
\mathrm{D}=1-\frac{\sum \mathrm{n}(\mathrm{n}-1)}{\mathrm{N}(\mathrm{N}-1)}
$$

where ' $\mathrm{D}$ ' represents diversity index, ' $N$ ' represents total number of species and ' $\mathrm{n}$ ' denotes number of a species of an individual.

Maturity index (MI): Maturity index for communities were measured with the method of Pichi-Sermolli (1948). 


$$
\text { Maturity Index }(\mathrm{MI})=\frac{\mathrm{Ft}}{\mathrm{N}}
$$

where ' $\mathrm{Ft}$ ' represents the frequency values of all species in a stand and ' $\mathrm{N}$ ' the total number of species in a stand.

\section{Cluster analysis}

PAST application and Principal Component Analysis (PCA) method were used to conduct cluster analysis. It is a classification method for grouping objects that are similar. The dendrogram that emerges is a hierarchical tree like structure. Different biotic communities can be represented by these cluster sampling units.

\section{Soil analysis}

The soil samples weighing about $1 \mathrm{~kg}$ were taken from the selected sites up to a depth of $15 \mathrm{~cm}$ of the area. To make a composite sample, the collected soil samples were thoroughly mixed and stored in polythene bags for physicochemical analysis. For the evaluation of physicochemical properties, the soil samples were analyzed at the Agriculture Research Institute Tarnab Peshawar. Soil texture triangle was used to assess the soil texture (Bouyoucos, 1936; Brady, 1990). The organic matter content was determined using the Walkley (1947) standard process, while $\mathrm{pH}$ of the soil was measured by using a portable PHS-25 pH meter (Black, 1965). Following acid-base neutralization, $\mathrm{CaCO}_{3}$ was determined (Rayan et al., 1997). Kjeldahl method (1983) was used to calculate nitrogen content. The concentration sodium $(\mathrm{Na})$ was calculated by using flame photometry, while the concentration of potassium (K) was determined through Olsen and Sommers's process (1982).

\section{Results}

\section{Family importance values}

The present study was conducted in Pashat valley along Pak-Afghan Border District Bajaur. Five monitoring sites viz. Bagandel, Batwar, Gabar, Saro Wano and Mala Said were recognized. The floristic composition and vegetation structure of the study area is dynamic. In total, 110 plants were identified in sampling units, with 23 species of trees, 32 species of shrubs and 55 species of herbs (Table 1). On the basis of importance value (IV), three communities each for trees, shrubs and herbs were documented in the monitoring sites. Seventeen families established the trees communities which is led by family Fagaceae with FIV 249.60 flowed by Moraceae (218.82), Oleaceae (148.95), Pinaceae (146.07), Juglandaceae (120.0), Simaroubaceae (102.27) and the rest of families having FIV value less than 100. Twenty (20) families represent shrubby communities, with Rosaceae dominating with FIV of 185.83, followed by Rhamnaceae (FIV 160.05), Lamiaceae (FIV 142.29), Berberidaceae (FIV 134.40), Papilionaceae (FIV 132.39) Buxaceae (FIV 112. 89) and Acanthaceae (FIV 110.79) while the remaining families have FIV ranges from 85.74 to minimum of 7.08. Similarly, herbaceous families were recorded 25 in which dominant families were Poaceae with FIV (399.28) followed by Lamiaceae (354.30) and Asteraceae (179.67) as shown in Table 1 and 2. 
Table 1. IV for trees, shrubs and herbs species in Pashat valley

\begin{tabular}{|c|c|c|c|c|c|c|}
\hline S\# & Species & $\begin{array}{c}\text { Site-I } \\
\text { BT }\end{array}$ & $\begin{array}{c}\text { Site-II } \\
\text { BG }\end{array}$ & \begin{tabular}{|c|} 
Site-III \\
GB
\end{tabular} & \begin{tabular}{|c} 
Site-IV \\
SW
\end{tabular} & \begin{tabular}{|c|} 
Site-V \\
MS
\end{tabular} \\
\hline \multicolumn{7}{|c|}{ Trees } \\
\hline 1 & Acacia modesta Wall. & 9.93 & 15.54 & 0 & 16.23 & 21.21 \\
\hline 2 & Ailanthus altissima (Mill.) Swingle & 13.38 & 17.13 & 19.62 & 22.89 & 29.25 \\
\hline 3 & Alnus nitida (Spach) Endl. Gen. & 0 & 0 & 16.47 & 0 & 0 \\
\hline 4 & Celtis aeriocarpa Decne. & 8.94 & 11.58 & 0 & 0 & 0 \\
\hline 5 & Celtis caucasica Wild. & 0 & 10.05 & 0 & 0 & 0 \\
\hline 6 & Diospyros lotus L. & 21.51 & 16.68 & 0 & 0 & 0 \\
\hline 7 & Ficus palmata Forssk. & 16.65 & 0 & 19.83 & 20.64 & 32.79 \\
\hline 8 & Juglans regia $\mathrm{L}$. & 23.64 & 17.31 & 20.04 & 28.53 & 30.48 \\
\hline 9 & Melia azedarach $\mathrm{L}$. & 17.88 & 0 & 10.05 & 17.28 & 21.48 \\
\hline 10 & Morus alba $\mathrm{L}$. & 15.69 & 15.15 & 24.12 & 0 & 22.05 \\
\hline 11 & Morus nigra $\mathrm{L}$. & 9.54 & 14.58 & 19.86 & 0 & 0 \\
\hline 12 & Olea ferruginea Royle. & 21.24 & 34.23 & 39.87 & 28.56 & 32.97 \\
\hline 13 & Pinus roxburghii Sargent & 16.59 & 19.47 & 0 & 16.11 & 0 \\
\hline 14 & Pinus wallichiana A.B. Jackson. & 39.81 & 54.09 & 0 & 0 & 0 \\
\hline 15 & Platanus orientalis L. & 19.14 & 15.24 & 22.29 & 0 & 0 \\
\hline 16 & Pyrus pashia Ham. ex D. D on. & 16.41 & 0 & 0 & 0 & 0 \\
\hline 17 & Quercus baloot Griffith & 0 & 0 & 27.57 & 22.56 & 0 \\
\hline 18 & Quercus dilatata A. Kern. & 0 & 0 & 0 & 21.63 & 0 \\
\hline 19 & Quercus incana Roxb., Hort. Beng. & 30.51 & 20.34 & 31.17 & 48.57 & 47.25 \\
\hline 20 & Robinia pseudoacacia $\mathrm{L}$. & 0 & 0 & 17.79 & 13.89 & 21.78 \\
\hline 21 & Salix acmophylla Boiss. & 0 & 13.59 & 16.68 & 15.45 & 20.22 \\
\hline 22 & Zanthoxylum armatum DC & 19.14 & 16.56 & 0 & 27.75 & 0 \\
\hline 23 & Ziziphus jujuba Mill. & 0 & 8.43 & 14.64 & 0 & 20.49 \\
\hline \multicolumn{7}{|c|}{ Shrubs } \\
\hline 24 & Andrachne cordifolia $\mathrm{L}$. & 11.88 & 19.65 & 12.24 & 0 & 0 \\
\hline 25 & Berberis lycium Royle & 30.09 & 32.7 & 22.32 & 20.04 & 29.25 \\
\hline 26 & Buddleja crispa Bth. & 23.73 & 0 & 0 & 17.58 & 20.79 \\
\hline 27 & Calotropis procera (Ait.) Ait.f., Hort. & 0 & 0 & 7.26 & 11.46 & 0 \\
\hline 28 & Capparis spinosa $\mathrm{L}$. & 0 & 0 & 0 & 0 & 7.08 \\
\hline 29 & Cestrum elegans (Brongn. ex Neumann) Schltdl. & 0 & 0 & 10.98 & 0 & 0 \\
\hline 30 & Cotoneaster microphyllus Wall. ex Lindl. & 13.35 & 15.21 & 10.83 & 12.42 & 15.06 \\
\hline 31 & Daphne mucronata Royle & 15.81 & 0 & 18.36 & 18.9 & 21.96 \\
\hline 32 & Daphne papyracea Wall. ex Steud., Nom. & 8.4 & 0 & 0 & 0 & 0 \\
\hline 33 & Desmodium elegans DC. & 9.3 & 16.38 & 0 & 0 & 0 \\
\hline 34 & Dodonaea viscosa (L.) Jacq. & 0 & 13.02 & 0 & 18.06 & 20.64 \\
\hline 35 & Hedera nepalensis K. Koch & 9.06 & 8.85 & 9.48 & 0 & 10.56 \\
\hline 36 & Indigofera heterantha Wall. ex Brandis. & 28.56 & 18.48 & 19.32 & 17.19 & 23.16 \\
\hline 37 & Jasminum humile $\mathrm{L}$. & 0 & 0 & 13.71 & 0 & 0 \\
\hline 38 & Jasminum officinale $\mathrm{L}$. & 10.53 & 0 & 0 & 0 & 0 \\
\hline 39 & Justicia adhatoda L. & 16.59 & 17.07 & 20.82 & 32.1 & 24.21 \\
\hline 40 & Lantana indica Roxb. & 0 & 0 & 0 & 11.73 & 14.16 \\
\hline 41 & Mallotus philippensis (Lam.) Muell. & 0 & 0 & 0 & 10.23 & 0 \\
\hline 42 & Maytenus royleanus Wall. ex Lawson & 10.32 & 0 & 0 & 0 & 0 \\
\hline 43 & Otostegia limbata (Bth.) Boiss & 19.71 & 32.25 & 26.85 & 26.67 & 36.81 \\
\hline 44 & Reinwardtia trigyna (Roxb.) Planch. & 0 & 11.85 & 18.99 & 0 & 0 \\
\hline 45 & Ricinus communis $\mathrm{L}$. & 0 & 0 & 12.21 & 10.71 & 0 \\
\hline 46 & Rubus ellipticus (Franch.) Thuan. & 0 & 6.99 & 17.19 & 0 & 0 \\
\hline 47 & Rubus fruticosus $\mathrm{L}$. & 18.15 & 18.66 & 30.3 & 13.35 & 0 \\
\hline 48 & Rubus niveus Thunb. & 13.92 & 0 & 0 & 0 & 0 \\
\hline 49 & Sageretia thea (Osbeck) M. C. Johnston & 17.91 & 17.34 & 21.45 & 16.05 & 25.41 \\
\hline 50 & Sarcococca saligna (D. Don) Muell. & 22.32 & 23.88 & 0 & 41.28 & 18.72 \\
\hline
\end{tabular}




\begin{tabular}{|c|c|c|c|c|c|c|}
\hline S\# & Species & $\begin{array}{c}\text { Site-I } \\
\text { BT }\end{array}$ & $\begin{array}{c}\text { Site-II } \\
\text { BG }\end{array}$ & $\begin{array}{c}\text { Site-III } \\
\text { GB }\end{array}$ & $\begin{array}{c}\text { Site-IV } \\
\text { SW }\end{array}$ & $\begin{array}{c}\text { Site-V } \\
\text { MS }\end{array}$ \\
\hline 51 & Verbena officinalis L. & 0 & 11.19 & 0 & 0 & 0 \\
\hline 52 & Viburnum grandiflorum Wall. ex Dc. & 0 & 9.03 & 0 & 0 & 0 \\
\hline 53 & Vitex negundo L. & 9.96 & 12.75 & 0 & 8.97 & 16.98 \\
\hline 54 & Withania somnifera (L.) Dunal. & 0 & 0 & 12.69 & 0 & 0 \\
\hline 55 & Ziziphus oxyphylla Edgew. & 10.41 & 14.7 & 15 & 13.26 & 15.21 \\
\hline \multicolumn{7}{|c|}{ Herbs } \\
\hline 56 & Achyranthes bidentata Blume & 0 & 0 & 5.19 & 6.25 & 0 \\
\hline 57 & Ajuga bracteosa Wall. ex Bth. & 10.69 & 10.58 & 8.97 & 11.6 & 0 \\
\hline 58 & Androsace rotundifolia Hardw. & 0 & 0 & 9.79 & 0 & 0 \\
\hline 59 & Apluda mutica $\mathrm{L}$. & 0 & 15.87 & 16.8 & 0 & 37.56 \\
\hline 60 & Argyrolobium roseum (Camb.) Jaub. & 0 & 0 & 6.99 & 0 & 0 \\
\hline 61 & Arisaema flavum (Forsk.) Schott, Prodr. & 6.14 & 6.95 & 0 & 0 & 0 \\
\hline 62 & Asparagus gracilis Royle. & 0 & 3.52 & 0 & 4.99 & 0 \\
\hline 63 & Bromus pectinatus Thunb. Prodr. Fl. Cap. & 0 & 0 & 0 & 0 & 9.44 \\
\hline 64 & Cannabis sativa $\mathrm{L}$. & 0 & 0 & 11.29 & 0 & 13.78 \\
\hline 65 & Carduus edelbergii Reh. F., K. Danske & 0 & 0 & 10.19 & 0 & 0 \\
\hline 66 & Chrysopogon serrulatus Trin. & 0 & 0 & 0 & 0 & 26.68 \\
\hline 67 & Cirsium vulgare (Savi) Ten. & 15.9 & 0 & 0 & 15.61 & 0 \\
\hline 68 & Clematis graveolens Lindl. & 0 & 0 & 9.08 & 0 & 0 \\
\hline 69 & Conyza canadensis (L.) Cronquist. & 15.5 & 13.63 & 12.22 & 24.61 & 20.26 \\
\hline 70 & Cynodon dactylon (L.) Pers. & 15.94 & 18.69 & 28.7 & 26.51 & 31.4 \\
\hline 71 & Cyperus niveus Retz., Observ. & 13.12 & 10.61 & 19.82 & 11.78 & 13.89 \\
\hline 72 & Delphinium ajacis (L.) Schur & 0 & 10.59 & 0 & 0 & 0 \\
\hline 73 & Dicliptera bupleuroides Nees & 0 & 0 & 0 & 10.83 & 0 \\
\hline 74 & Duchesnea indica (Jacks.) Focke & 7.37 & 9.56 & 8.65 & 7.33 & 9.05 \\
\hline 75 & Eragrostis minor Host & 12.14 & 0 & 0 & 13.66 & 0 \\
\hline 76 & Eragrostis papposa (Roem \& Schult.) Steud. Nom. & 0 & 16.65 & 0 & 0 & 0 \\
\hline 77 & Erioscirpus comosus (Wall.) Palla & 0 & 0 & 0 & 9.64 & 0 \\
\hline 78 & Filago hurdwarica (Wall. ex DC) Wagenitz & 0 & 0 & 0 & 0 & 13.04 \\
\hline 79 & Filago pyramidata $\mathrm{L}$. & 0 & 8.62 & 0 & 7.12 & 0 \\
\hline 80 & Fimbristylis cymosa R. Br., Prodr. & 0 & 0 & 5.19 & 0 & 0 \\
\hline 81 & Geranium rotundifolium $\mathrm{L}$. & 0 & 0 & 8.38 & 15.28 & 0 \\
\hline 82 & Herniaria hirsuta $\mathrm{L}$. & 0 & 0 & 6.34 & 0 & 0 \\
\hline 83 & $\begin{array}{l}\text { Heteropogon contortus (L.) P. Beauy. ex. Roem \& } \\
\text { Schult. }\end{array}$ & 11.79 & 17.81 & 0 & 0 & 0 \\
\hline 84 & Hyparrhenia hirta (L.) Stapf & 16.23 & 21.56 & 0 & 12.27 & 0 \\
\hline 85 & Impatiens edgeworthii Hook. f., Fl. Brit. & 0 & 0 & 0 & 11.82 & 0 \\
\hline 86 & Marrubium vulgare $\mathrm{L}$. & 0 & 0 & 0 & 15.63 & 10.37 \\
\hline 87 & Medicago minima (L.) Grub. & 0 & 0 & 9.38 & 0 & 0 \\
\hline 88 & Mentha longifolia $\mathrm{L}$. & 0 & 0 & 7.03 & 0 & 0 \\
\hline 89 & Micromeria biflora (Buch. Ham. Ex. D. Don) Bth. & 13.18 & 9.83 & 10.94 & 10.6 & 12.86 \\
\hline 90 & Nepeta cataria $\mathrm{L}$. & 8.145 & 8.69 & 7.22 & 10.74 & 9.31 \\
\hline 91 & Origanum vulgare $\mathrm{L}$. & 15.8 & 0 & 0 & 0 & 0 \\
\hline 92 & Oxalis corniculata $\mathrm{L}$. & 13.84 & 13.16 & 17.38 & 12.66 & 14.1 \\
\hline 93 & Pennisetum orientale Rich. & 15.1 & 13.71 & 0 & 0 & 0 \\
\hline 94 & Phalaris paradoxa $\mathrm{L}$. & 0 & 0 & 0 & 8.183 & 0 \\
\hline 95 & Plantago lanceolata $\mathrm{L}$. & 11.96 & 0 & 0 & 0 & 12.79 \\
\hline 96 & Pteris cretica $\mathrm{L}$. & 9.92 & 0 & 0 & 0 & 8.77 \\
\hline 97 & Rhynchosia minima (L.) DC. & 0 & 5.51 & 0 & 0 & 0 \\
\hline 98 & Rubia cordifolia $\mathrm{L}$. & 0 & 13.18 & 8.52 & 0 & 0 \\
\hline 99 & Rumex hastatus D. Don. & 23.92 & 12.57 & 13.13 & 0 & 0 \\
\hline 100 & Salvia moorcroftiana Wall. ex Bth. & 0 & 0 & 19.84 & 0 & 0 \\
\hline 101 & Stachys parviflora Bth. & 0 & 0 & 0 & 16.19 & 16.56 \\
\hline 102 & Tagetes minuta $\mathrm{L}$. & 0 & 11.71 & 11.26 & 0 & 0 \\
\hline
\end{tabular}




\begin{tabular}{c|c|c|c|c|c|c}
\hline S\# & Species & $\begin{array}{c}\text { Site-I } \\
\text { BT }\end{array}$ & $\begin{array}{c}\text { Site-II } \\
\text { BG }\end{array}$ & $\begin{array}{c}\text { Site-III } \\
\text { GB }\end{array}$ & $\begin{array}{c}\text { Site-IV } \\
\text { SW }\end{array}$ & $\begin{array}{c}\text { Site-V } \\
\text { MS }\end{array}$ \\
\hline 103 & Tetrapogon villosus Desf. & 0 & 12.59 & 0 & 0 & 0 \\
104 & Teucrium royleanum Wall. ex Bth & 15.86 & 0 & 11.65 & 0 & 15.13 \\
105 & Thymus linearis Bth. ex Wall. & 14.62 & 10.44 & 7.26 & 23.28 & 11.3 \\
106 & Tulipa clusiana DC. & 12 & 0 & 0 & 0 & 13.7 \\
107 & Urtica dioica L. & 0 & 8.49 & 0 & 0 & 0 \\
108 & Verbascum thapsus L. & 11.12 & 7.89 & 8.77 & 0 & 0 \\
109 & Viola canescens Wall. ex Roxb. & 0 & 7.57 & 0 & 13.39 & 0 \\
110 & Viola pilosa Blume & 9.73 & 0 & 0 & 0 & 0 \\
\hline
\end{tabular}

Key: BT-Batwar, BG- Bagandel, GB-Gabar, SW- Saro Wano, MS- Mala Said

Table 2. Family importance values of Pashat valley

\begin{tabular}{|c|c|c|c|c|c|}
\hline S\# & Family & FIV & S\# & Family & FIV \\
\hline \multicolumn{3}{|c|}{ Trees } & \multicolumn{3}{|c|}{ Shrubs } \\
\hline 1 & Fagaceae & 249.6 & 15 & Oleaceae & 24.24 \\
\hline 2 & Moraceae & 218.82 & 16 & Solanaceae & 23.67 \\
\hline 3 & Oleaceae & 148.95 & 17 & Asclepiadaceae & 18.72 \\
\hline 4 & Pinaceae & 146.07 & 18 & Celastraceae & 10.32 \\
\hline 5 & Juglandaceae & 120 & 19 & Caprifoliaceae & 9.03 \\
\hline 6 & Simaroubaceae & 102.27 & 20 & Capparidaceae & 7.08 \\
\hline 7 & Meliaceae & 66.69 & \multicolumn{3}{|c|}{ Herbs } \\
\hline 8 & Salicaceae & 65.94 & 1 & Poaceae & 399.28 \\
\hline 9 & Rutaceae & 63.45 & 2 & Lamiaceae & 354.3 \\
\hline 10 & Mimosaceae & 62.91 & 3 & Asteraceae & 179.67 \\
\hline 11 & Platanaceae & 56.67 & 4 & Cyperaceae & 84.05 \\
\hline 12 & Papilionaceae & 53.46 & 5 & Oxalidaceae & 71.14 \\
\hline 13 & Rhamnaceae & 43.56 & 6 & Polygonaceae & 49.62 \\
\hline 14 & Ebenaceae & 38.19 & 7 & Rosaceae & 41.96 \\
\hline 15 & Ulmaceae & 30.57 & 8 & Violaceae & 30.69 \\
\hline 16 & Betulaceae & 16.47 & 9 & Scrophulariaceae & 27.79 \\
\hline 17 & Rosaceae & 16.41 & 10 & Liliaceae & 25.7 \\
\hline \multicolumn{3}{|c|}{ Shrubs } & 11 & Cannabaceae & 25.07 \\
\hline 1 & Rosaceae & 185.43 & 12 & Plantaginaceae & 24.75 \\
\hline 2 & Rhamnaceae & 160.05 & 13 & Geraniaceae & 23.66 \\
\hline 3 & Lamiaceae & 142.29 & 14 & Papilionaceae & 21.88 \\
\hline 4 & Berberidaceae & 134.4 & 15 & Rubiaceae & 21.69 \\
\hline 5 & Papilionaceae & 132.39 & 16 & Ranunculaceae & 19.67 \\
\hline 6 & Buxaceae & 112.89 & 17 & Pteridaceae & 18.69 \\
\hline 7 & Acanthaceae & 110.79 & 18 & Aracaceae & 13.08 \\
\hline 8 & Verbenaceae & 85.74 & 19 & Balsaminaceae & 11.82 \\
\hline 9 & Thymelaeaceae & 83.43 & 20 & Amaranthaceae & 11.45 \\
\hline 10 & Euphorbiaceae & 76.92 & 21 & Acanthaceae & 10.83 \\
\hline 11 & Buddlejaceae & 62.1 & 22 & Primulaceae & 9.79 \\
\hline 12 & Sapindaceae & 51.72 & 23 & Asparagaceae & 8.52 \\
\hline 13 & Araliaceae & 37.95 & 24 & Urticaceae & 8.49 \\
\hline 14 & Linaceae & 30.84 & 25 & Illecebraceae & 6.34 \\
\hline
\end{tabular}




\section{Communities structure at the monitoring sites}

\section{Site I (Batwar)}

Plant communities were established at elevation of $2002 \mathrm{~m}$ in the North-East of the valley at $34.56^{\circ} \mathrm{N}$ latitude and $71.29^{\circ} \mathrm{E}$ longitude. The soil texture was silty, clay loam with $54 \%$ silt, $32 \%$ clay and $14 \%$ sand. The $\mathrm{pH}$ of site recorded as 7.4 . The soil had nitrogen content of $0.036 \mathrm{mg} / \mathrm{kg}$, phosphorus content of $15.4 \mathrm{mg} / \mathrm{kg}$, and a potassium content of $80 \mathrm{mg} / \mathrm{kg}$. The electrical conductivity was recorded $0.01 \%$ and the total soluble salts were $0.19 \mathrm{dsm}^{-1}$. The $\mathrm{CaCO}_{3}$ content was $9.0 \%$, with just $0.72 \%$ organic matter (Table 3).

\section{Pinus-Quercus-Juglans community (PQJ)}

Pinus wallichiana dominated the community with an IV of 39.82 which is associated with Quercus incana with IV of 30.51 and Juglans regia with an IV 23.66. These were followed by Diospyrus lotus (IV 21.51), Olea ferruginea (IV 21.24), Platanus orientalis (IV 19.16) and Zanthoxylum armatum (IV 19.14). In addition to these, Melia azedarach, Ficus palmata, Pinus roxburghii, Pyrus pashia, Morus alba, Ailanthus altissimia, Acacia modesta, Morus nigra and Celtis aeropcarpa had IV value ranging from 17.87 to 8.94 (Table 1).

\section{Berberis-Indigofera-Buddleja community (BIB)}

Berberis lyceum, with an IV of 30.10 uniformly dominated this community. Indigofera heterantha with an IV of 28.55, and Buddleja crispa with an IV of 23.71, were the other dominant species. Sarcococca saligna (IV of 22.30) form mosaic patches in the community due to soil type and topographical variation. The co-dominant species were Otostegia lambata (19.73 IV) followed by Rubus fruticosa (18.16 IV), Sageretia thea (17.92 IV) and Justicia adhatoda (16.59 IV). Daphne mucronata, Rubus niveus, Cotoneaster microphyllus, Andrachne cordifolia, Jasminum officinale, Maytenus royleanus, Vitex negundo, Desmodium elegans, Hedera nepalensis and Daphne papyracea were the other shrubby members of the community with IV ranges from 15.80 to 8.40 (Table 1).

\section{Rumex-Hyparrhenia-Cynodon community (RHC)}

This community consists of 23 species led by Rumex hastatus (IV 23.92) followed by Hyparrhenia hirta (IV 16.23), Cynodon dactylon (IV 15.94), Cirsium vulgare (IV 15.90), Teucrium royleanum (IV 15.86), Origanum vulgare (IV 15.80), Conyza Canadensis (IV 15.50) and Pennisetum orientale (IV 15.10). The remaining species in the community had low IV value ranged from 14.62 to 6.14 (Table1).

\section{Site II (Bagandel)}

At this site, the plant community established on North and East aspect with elevation of $1895 \mathrm{~m}$ at $34.57^{\circ} \mathrm{N}$ latitude and $71.32^{\circ} \mathrm{E}$ longitude. The texture class composed of $52 \%$ silt, $32 \%$ clay and $16 \%$ sand. The $\mathrm{pH}$ of site recorded as 7.8 . The soil had nitrogen content of $0.043 \mathrm{mg} / \mathrm{kg}$, phosphorus content of $14.3 \mathrm{mg} / \mathrm{kg}$ and potassium content of $90 \mathrm{mg} / \mathrm{kg}$. Total soluble salts (TSS) were recorded $0.064 \%$ and the electrical conductivity (EC) was $0.02 \mathrm{dsm}^{-1}$. The $\mathrm{CaCO}_{3}$ content was $8.5 \%$ with low organic matter $0.86 \%$ (Table 3). 
Table 3. Physicochemical analysis of soil samples of the selected sites of the Pashat valley

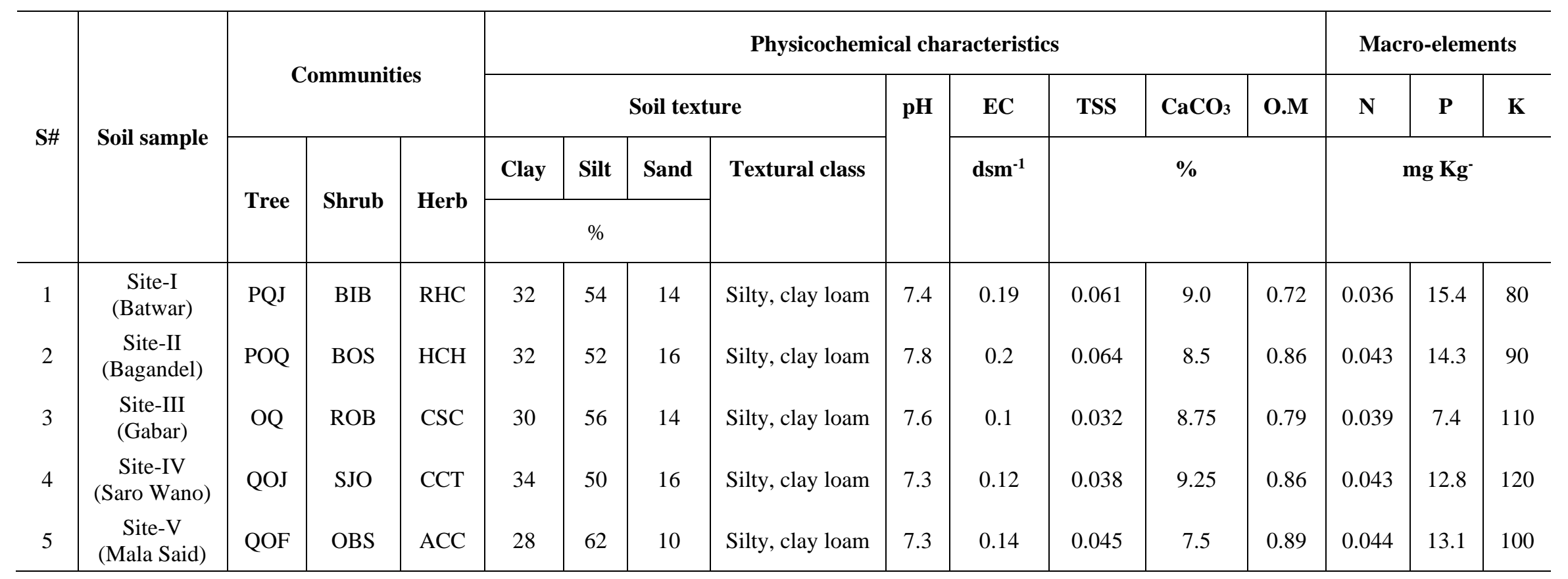

Key: E.C= Electrical conductivity, TSS= Total Soluble Salts, O.M= Organic matter, N= Nitrogen, $\mathrm{P}=$ Phosphorus, $\mathrm{K}=\mathrm{Potassium}$

Key for communities:

Tree: $\mathrm{PQJ}=$ Pinus-Quercus-Juglans, $\mathrm{POQ}=$ Pinus-Olea-Quercus, $\mathrm{OQ}=$ Olea-Quercus, QFJ=Quercus-Olea-Juglans, QOF= Quercus-Olea-Ficus

Shrubs: $\mathrm{BIB}=$ Berberis-Indigofera-Buddleja, $\mathrm{BOS}=$ Berberis-Otostegia-Sarcococca, $\mathrm{ROB}=$ Rubus-Otostegia-Berberis, $\mathrm{SJO}=$ Sarcococca- Justicia-Otostegia, $\mathrm{OBS}=$ Otostegia-Berberis-Sageretia

Herb: $\mathrm{RHC}=$ Rumex - Hyparrhenia-Cynodon, $\mathrm{HCH}=$ Hyparrhenia-Cynodon-Heteropogon, $\mathrm{CSC}=$ Cynodon-Salvia-Cyperus, $\mathrm{CCT}=$ Cynodon-Conyza-Thymus, $\mathrm{ACC}=$ Apluda-Cynodon-Chrysopogon 


\section{Pinus-Olea-Quercus community (POQ)}

Tree community at site II was dominated by Pinus wallichiana with an IV of 54.08. This was followed by Olea ferruginea (IV 34.24), next by Quercus incana (IV 20.23). Pinus roxburghii (IV 19.48), Juglans regia (IV 17.33), Ailanthus altissima (IV 17.11) and Diospyros lotus (IV 16.67) were the next co-dominant plants in the list. In addition to these, Zanthoxylum armatum, Acacia modesta, Platanus orientalis, Morus alba, Morus nigra, Salix acmophylla, Celtis aeriocarpa, Celtis caucasica and Ziziphus jujuba had IV value ranging from 16.54 to 8.42 (Table1).

\section{Berberis-Otostegia-Sarcococca community (BOS)}

Berberis lyceum with an IV of 32.71 was found dominant with uniform distribution in the community. The other dominant species were Otostegia limbata with an IV of 32.25 and Sarcococca saligna of 23.87. The other associated species were Andrachne cordifolia (19.67 IV) followed by Rubus fruticosa (18.64 IV), Indigofera heterantha (18.49 IV) and Sageretia thea (17.34 IV). Other shrubby members of the community were Justicia adhatoda, Desmodium elegans, Cotoneaster microphyllus, Ziziphus oxyphylla, Dodonaea viscosa, Vitex negundo, Reinwardtia trigyna, Desmodium elegans, Verbena officinalis, Viburnum grandiflorum, Hedera nepalensis and Rubus ellipticus had IV between 17.09 and 6.99 (Table1).

\section{Hyparrhenia-Cynodon-Heteropogon community $(\mathrm{HCH})$}

Grass species were found dominant in this community on the basis of importance values such as Hyparrhenia hirta (IV 21.56), Cynodon dactylon (IV 18.69) and Heteropogon contortus (IV 17.81). The other co-dominant species were Eragrostis papposa (16.65), Apluda mutica (15.87), Pennisetum orientale, (13.71), Conyza Canadensis (13.63) and Rubia cordifolia (13.18) (Table 1).

\section{Site III (Gabber)}

Communities at this site were documented at the North aspect at low elevation of $1553 \mathrm{~m}$ at $34.54^{\circ} \mathrm{N}$ latitude and $71.29^{\circ} \mathrm{E}$ longitude. The texture class was composed of $56 \%$ silt, $30 \%$ clay and $14 \%$ sand. The $\mathrm{pH}$ of site recorded as 7.6. Soil had nitrogen content $0.039 \mathrm{mg} / \mathrm{kg}$, phosphorus $7.4 \mathrm{mg} / \mathrm{kg}$ and potassium $110 \mathrm{mg} / \mathrm{kg}$. The electrical conductivity was recorded $0.10 \mathrm{dsm}^{-1}$ and the total soluble salts (TSS) content $0.032 \%$. $\mathrm{CacO}_{3}$ content was $8.75 \%$ with just $0.79 \%$ organic matter (Table 3 ).

\section{Olea-Quercus community (OQ)}

Olea ferruginea was found to be dominated with IV of 39.86. Other associated dominant species were Quercus incana having an IV of 31.17 and Quercus baloot with an IV 27.59. These were followed by Morus alba (IV 24.11), Platanus orientalis (IV 22.30) and Juglans regia (IV 20.05). In addition to these Morus nigra, Ficus palmata, Ailanthus altissima, Robinia pseudoacacia, Salix acmophylla, Alnus nitida, Ziziphus jujube and Melia azedarach had IV value ranging from 19.86 to 10.04 (Table1).

\section{Rubus-Otostegia-Berberis community (ROB)}

This community was led by Rubus fruticosus with an IV of 30.30. The other dominant species were Otostegia limbata with an IV of 26.86 and Berberis lyceum of 22.33. The 


$$
-3590-
$$

other associated species were Sageretia thea (21.45 IV) followed by Justicia adhatoda (20.81 IV), Indigofera heterantha (19.33 IV) and Reinwardtia trigyna (18.98 IV). Other members of the community were Daphne mucronata, Rubus ellipticus, Ziziphus oxyphylla, Jasminum humile, Withania somnifera, Andrachne cordifolia, Ricinus communis, Cestrum elegans, Cotoneaster microphyllus, Hedera nepalensis and Capparis spinosa had IV ranging from 18.37 to 7.26 .

\section{Cynodon-Salvia-Cyperus community (CSC)}

Cynodon dactylon (IV 28.70), Salvia moorcroftiana (IV 19.84) and Cyperus niveus (IV 19.82) were the leading representative species of the community. The next dominant species in this community were Oxalis corniculata with IV (17.38), Apluda mutica (16.80), Rumex hastatus (13.13), Conyza Canadensis (12.22) and Teucrium royleanum (11.65) (Table 1).

\section{Site IV (Saro Wano)}

This community was established at $1756 \mathrm{~m}$ elevation in the North-West aspect of the study area at $34.53^{\circ} \mathrm{N}$ latitude and $71.27^{\circ} \mathrm{E}$ longitude. The soil texture class was composed of $50 \%$ silt, $34 \%$ clay and $16 \%$ sand. The $\mathrm{pH}$ of site recorded as 7.3 . Soil nitrogen content was estimated $(0.043 \mathrm{mg} / \mathrm{kg})$, phosphorus $(12.8 \mathrm{mg} / \mathrm{kg})$ and potassium $\left(120 \mathrm{mg} / \mathrm{kg}\right.$ ). The electrical conductivity was recorded $0.12 \mathrm{dsm}^{-1}$ and total soluble salts (TSS) $0.038 \% . \mathrm{CacO}_{3}$ content was $9.25 \%$ with low organic matter $0.86 \%$ (Table 3).

\section{Quercus-Ficus-Juglans community (QFJ)}

Quercus incana was found to be dominated by IV of 48.57. Other accompanied dominant species were Ficus palmata with IV of 28.55 and Juglans regia with an IV 28.53. They were followed by Zanthoxylum armatum (IV 27.74), Ailanthus altissima (IV 22.89) and Quercus baloot (IV 22.54). In addition to these Quercus dilatata, Olea ferruginea, Melia azedarach, Acacia modesta, Pinus roxburghii, Salix acmophylla and Robinia pseudoacacia had IV value ranging from 21.60 to 13.04 (Table1).

\section{Sarcococca-Justicia-Otostegia community (SJO)}

Sarcococca saligna dominated this community uniformly with an IV of 41.29. The other dominant species were Justicia adhatoda with an IV of 32.10 and Otostegia limbata of 26.68. Berberis lycium (20.06 IV) followed by Daphne mucronata (18.91 IV), Dodonaea viscosa (18.05 IV) and Buddleja crispa (17.58 IV) were the other dominant species. Other shrubs within the community were Indigofera heterantha, Sageretia thea, Rubus fruticosus, Ziziphus oxyphylla, Cotoneaster microphyllus, Lantana indica, Calotropis procera, Ricinus communis, Mallotus philippensis, and Vitex negundo had IV between 17.19 and 8.97 (Table1).

\section{Cynodon-Conyza-Thymus community (CCT)}

The leading species of this community were Cynodon dactylon with an IV of 26.51, Conyza canadensis with an IV of 24.61, and Thymus linearis with an IV of 23.28. Stachys parviflora (16.19), Marrubium vulgare (15.63), Cirsium vulgare (15.61), Geranium rotundifolium (15.28) and Eragrostis minor (13.66) were the other co-dominant species in the site (Table 1). 


\section{Site V(Mala Said)}

The plant communities at this site are established at West aspect at $34.51^{\circ} \mathrm{N}$ latitude and $71.28^{\circ} \mathrm{E}$ longitude with an elevation of $1805 \mathrm{~m}$. The soil texture class was composed of $62 \%$ silt, $28 \%$ clay and $10 \%$ sand. The $\mathrm{pH}$ of site recorded as 7.3 . The calculated macro-element such as nitrogen was $0.044 \mathrm{mg} / \mathrm{kg}$, phosphorus $13.1 \mathrm{mg} / \mathrm{kg}$ and potassium $100 \mathrm{mg} / \mathrm{kg}$. The organic matter content was $0.89 \%$ and $\mathrm{CacO}_{3} 7.50 \%$. The total soluble salts (TSS) were recoded $0.045 \%$ and electrical conductivity $0.14 \mathrm{dsm}^{-1}$ (Table 3).

\section{Quercus-Olea-Ficus community (QOF)}

Quercus incana was found to be dominated with IV of 47.25. Other associated dominant species were Olea ferruginea having an IV of 32.97 and Ficus palmata with an IV 32.79. These were followed by Juglans regia (IV 30.4), Ailanthus altissima (IV 29.26) and Morus alba (IV 22.05). In addition to these Robinia pseudoacacia, Melia azedarach, Acacia modesta, Ziziphus jujuba, Salix acmophylla had IV value ranging from 21.78 to 20.22 (Table1).

\section{Otostegia-Berberis-Sageretia community (OBS)}

The dominant species in this community were Otostegia limbata (IV 36.81), Berberis lyceum (IV 29.24) and Sageretia thea (IV 25.40). Other co-exiting species in the community were Justicia adhatoda (24.20 IV) followed by Indigofera heterantha (23.17 IV), Daphne mucronata (21.95 IV) and Buddleja crispa (20.79 IV). Dodonaea viscosa, Sarcococca saligna, Vitex negundo, Ziziphus oxyphylla, Cotoneaster microphyllus, Lantana indica, Hedera nepalensis, and Capparis spinosa had IV between 20.63 and 7.09 were the other shrubby members of the community (Table 1).

\section{Apluda-Cynodon-Chrysopogon community (ACC)}

This community was dominated by the grass species such as Apluda mutica (IV 37.56), Cynodon dactylon (IV 41.40) and Chrysopogon serrulatus (IV 26.68 IV). The next associated species were Conyza canadensis (20.26), Stachys parviflora (16.56), Teucrium royleanum (15.13), Oxalis corniculata (14.10) and Cyperus niveus (13.89) (Table 1).

\section{Index of Similarity (IS)}

\section{Tree communities}

The PQJ community of site I (Batwar) was found to be $6.84 \%$ similar to POQ community of Site II (Bagandel) as per Motyka's index of similarity (Motyka et al., 1950). The similarity between Batwar site I community i.e., PQJ and Gabar site III community i.e., OQ was found to be $11.05 \%$. Similarity between the community PQJ and QFJ at Saro Wano, site IV was $10.62 \%$. Similarly, the similarity index between PQJ and QOF communities in Mala Said, site V was $10.38 \%$. Similarity between Bagandel site II, tree community (POQ) and Gabar site III (OQ) was 7.86\%. POQ community resembled the tree community QOJ at Saro Wano, site IV by $10.59 \%$ and the QOF community at Mala Said, site V by $11.89 \%$. OQ tree community at Gabar site III showed $10.55 \%$ similarity with QFJ tree community at Saro Wano site IV, and $11.71 \%$ with QOF community at Mala Said site V. QFJ community of Saro Wano site IV shows $12.48 \%$ similarity with QOF community at Mala Said site V (Table 4). 
Table 4. Indices of similarity of tree communities

\begin{tabular}{c|ccccc}
\hline & PQJ & POQ & OQ & QOJ & QOF \\
\hline PQJ & $\mathrm{X}$ & $\mathrm{X}$ & $\mathrm{X}$ & $\mathrm{X}$ & $\mathrm{X}$ \\
POQ & 6.84 & $\mathrm{X}$ & $\mathrm{X}$ & $\mathrm{X}$ & $\mathrm{X}$ \\
OQ & 11.05 & 7.86 & $\mathrm{X}$ & $\mathrm{X}$ & $\mathrm{X}$ \\
QOJ & 8.49 & 10.59 & 10.55 & $\mathrm{X}$ & $\mathrm{X}$ \\
QOF & 10.38 & 11.89 & 11.71 & 12.48 & $\mathrm{X}$ \\
\hline
\end{tabular}

Key: $P Q J=$ Pinus- Quercus- Juglans, $P O Q=$ Pinus- Olea- Quercus, $O Q=$ Olea- Quercus, QFJ= QuercusOlea- Juglans, QOF= Quercus- Olea- Ficus

\section{Shrubby communities}

Motyka's index showed minimum similarities among the shrubby communities in the selected sites. The BIB community at site I (Batwar) was 5.79\%, similar with the BOS community at site II (Bagandel). At Gabar site III, BIB community was found to be $6.17 \%$, similar to the ROB community. At site IV (Saro Wano), there was a $6.32 \%$ similarity between the BIB and SJO communities. At Mala Said site V, the BIB community was $6.54 \%$, similar to OBS community. Bagandel site II, community BOS was $6.11 \%$ similar to ROB community at site III (Gabar). The BOS community of Bagandel site II was $7.24 \%$ similar to SJO community at Saro Wano site IV. At Mala Said site V, there was a $6.47 \%$ similarity between the BOS and OBS community. The shrub community ROB at Gabar site III was found $7.64 \%$ similar to the community SJO at Saro Wano site IV, while the OBS at site V (Mala Said) was found to be 5.94\% similar. The shrub community SJO at Saro Wano site IV and the OBS community at Mala Said site $\mathrm{V}$ had highest similarity of $8.63 \%$ (Table 5).

Table 5. Indices of similarity of shrubby communities

\begin{tabular}{c|ccccc}
\hline & BIB & BOS & ROB & SJO & OBS \\
\hline BIB & $\mathrm{X}$ & $\mathrm{X}$ & $\mathrm{X}$ & $\mathrm{X}$ & $\mathrm{X}$ \\
BOS & 5.97 & $\mathrm{X}$ & $\mathrm{X}$ & $\mathrm{X}$ & $\mathrm{X}$ \\
ROB & 6.17 & 6.11 & $\mathrm{X}$ & $\mathrm{X}$ & $\mathrm{X}$ \\
SJO & 6.32 & 7.24 & 7.64 & $\mathrm{X}$ & $\mathrm{X}$ \\
OBS & 6.54 & 6.47 & 5.94 & $\mathbf{8 . 6 3}$ & $\mathrm{X}$ \\
\hline
\end{tabular}

Key: $\mathrm{BIB}=$ Berberis- Indigofera- Buddleja, $\mathrm{BOS}=$ Berberis- Otostegia- Sarcococca, ROB $=$ RubusOtostegia- Berberis, $\mathrm{SJO}=$ Sarcococca- Justicia- Otostegia, OBS= Otostegia- Berberis- Sageretia

\section{Herbaceous communities}

RHC community at site I i.e., Batwar was $4.36 \%$ identical to $\mathrm{HCH}$ community at Bagandel site II among the herbaceous communities. The RHC community was found to be $5.12 \%$ similar to CSC community at Gabar site III, and $4.49 \%$ similar to the CCT community at Saro Wano site IV. RHC community was $2.97 \%$ similar to ACC community at Mala Said site V. The HCH community of Bagendel site II was $5.31 \%$ similar to CSC community at Gabar site III, $2.83 \%$ to CCT community at Saro Wano site IV and $7.47 \%$ to ACC community at Mala Said site V. The CSC herb community of Gabar site III was 3.82\% similar with CCT community at Saro Wano site IV and 6.19\% 
with ACC community at Mala Said site V. There was $5.46 \%$ similarity between the CCT community at Saro Wano site IV and the ACC community at Mala Said site V (Table 6).

Table 6. Indices of similarity of herbaceous communities

\begin{tabular}{c|ccccc}
\hline & RHC & HCH & CSC & CCT & ACC \\
\hline RHC & $\mathrm{X}$ & $\mathrm{X}$ & $\mathrm{X}$ & $\mathrm{X}$ & $\mathrm{X}$ \\
HCH & 4.36 & $\mathrm{X}$ & $\mathrm{X}$ & $\mathrm{X}$ & $\mathrm{X}$ \\
CSC & 5.12 & 5.31 & $\mathrm{X}$ & $\mathrm{X}$ & $\mathrm{X}$ \\
CCT & 4.49 & 2.83 & 3.82 & $\mathrm{X}$ & $\mathrm{X}$ \\
ACC & 4.97 & 7.47 & 6.19 & 5.46 & $\mathrm{X}$ \\
\hline
\end{tabular}

Key: $\mathrm{RHC}=$ Rumex- Hyparrhenia- Cynodon, $\mathrm{HCH}=$ Hyparrhenia- Cynodon- Heteropogon, $\mathrm{CSC}=$ Cynodon- Salvia- Cyperus, $\mathrm{CCT}=$ Cynodon- Conyza- Thymus, $\mathrm{ACC}=$ Apluda- Cynodon- Chrysopogon

\section{Species diversity $(S D)$}

Simpson' diversity was calculated for the tree communities at site I was 0.992 , site II 0.87 site III 0.937 , site IV 0.917 and site V 0.911 . Similarly, for shrubby community of site I and site II 0.937 each, site III 0.943, site IV 0.919 and site V 0.926 . The diversity index for herbaceous communities calculated from 0.906 to 0.961 (Table 7). Diversity of species was affected by various abiotic and biotic factors such as elevation, slope, erosion, soil texture, soil structure, climatic condition, grazing, browsing and anthropogenic activities in terms of deforestations. Species diversity is one of the greatest features of plants communities reflecting its structure and composition.

Table 7. Simpson's diversity indices among the various communities

\begin{tabular}{|c|c|c|c|}
\hline S\# & Sites & Communities & Simpson's diversity index \\
\hline \multicolumn{4}{|c|}{ Trees } \\
\hline 1 & Site-I & Pinus-Quercus-Juglans & 0.922 \\
\hline 2 & Site-II & Pinus-Olea-Quercus & 0.87 \\
\hline 3 & Site-III & Olea-Quercus- Quercus & 0.937 \\
\hline 4 & Site-IV & Quercus-Olea-Juglans & 0.917 \\
\hline 5 & Site-V & Quercus-Olea-Ficus & 0.911 \\
\hline \multicolumn{4}{|c|}{ Shrubs } \\
\hline 1 & Site-I & Berberis-Indigofera-Buddleja & 0.937 \\
\hline 2 & Site-II & Berberis-Otostegia-Sarcococca & 0.937 \\
\hline 3 & Site-III & Rubus-Otostegia-Berberis & 0.943 \\
\hline 4 & Site-IV & Sarcococca-Justicia-Otostegia & 0.919 \\
\hline 5 & Site-V & Otostegia-Berberis- Sageretia & 0.926 \\
\hline \multicolumn{4}{|c|}{ Herbs } \\
\hline 1 & Site-I & Rumex-Hyparrhenia-Cynodon & 0.957 \\
\hline 2 & Site-II & Hyparrhenia-Cynodon-Heteropogon & 0.961 \\
\hline 3 & Site-III & Cynodon-Salvia-Cyperus & 0.906 \\
\hline 4 & Site-IV & Cynodon-Conyza-Thymus & 0.951 \\
\hline 5 & Site-V & Apluda-Cynodon-Chrysopogon & 0.938 \\
\hline
\end{tabular}




\section{Maturity index (MI)}

The maturity index value for the trees, shrubs and herbs communities of the five selected sites ranged from 50.91 to 24.64. The highest maturity indices recorded for tree community at Mala Said site (50.91) and lowest for Bagandel site (36.25). The maturity indices for shrubby communities were highest for Mala Said site (46.0) and lowest for Batwar site (37.89). The herbaceous community at Bagandel site (37.18) had the highest maturity index, while Saro Wano Site (24.64) of the valley had the lowest value (Table 8). Value of low maturity indicates the heterogeneity in the plant communities due to its poor adaptation to environmental condition of the area.

Table 8. Maturity indices of tree, shrubby and herbaceous communities

\begin{tabular}{|c|c|c|c|}
\hline S\# & Sites & Communities & Maturity Index \\
\hline \multicolumn{4}{|c|}{ Trees } \\
\hline 1 & Site-I & Pinus-Quercus-Juglans & 48.75 \\
\hline 2 & Site-II & Pinus-Olea-Quercus & 36.25 \\
\hline 3 & Site-III & Olea-Quercus- Quercus & 47.14 \\
\hline 4 & Site-IV & Quercus-Olea-Juglans & 50.77 \\
\hline 5 & Site-V & Quercus-Olea-Ficus & 50.91 \\
\hline \multicolumn{4}{|c|}{ Shrubs } \\
\hline 1 & Site-I & Berberis-Indigofera-Buddleja & 37.89 \\
\hline 2 & Site-II & Berberis-Otostegia-Sarcococca & 39.44 \\
\hline 3 & Site-III & Rubus-Otostegia-Berberis & 40.55 \\
\hline 4 & Site-IV & Sarcococca-Justicia-Otostegia & 40.58 \\
\hline 5 & Site-V & Otostegia-Berberis- Sageretia & 46.0 \\
\hline \multicolumn{4}{|c|}{ Herbs } \\
\hline 1 & Site-I & Rumex-Hyparrhenia-Cynodon & 29.27 \\
\hline 2 & Site-II & Hyparrhenia-Cynodon-Heteropogon & 37.18 \\
\hline 3 & Site-III & Cynodon-Salvia-Cyperus & 28.39 \\
\hline 4 & Site-IV & Cynodon-Conyza-Thymus & 24.64 \\
\hline 5 & Site-V & Apluda-Cynodon-Chrysopogon & 30.17 \\
\hline
\end{tabular}

\section{Cluster analysis using PAST}

Data sets for 110 recorded species across 150 quadrats of five selected sites, classified the plants into four different groups via cluster analysis using PAST software version 4.03 using quantitative values based on the combined IV of each species.

Cluster 1. This group was dominated by tree and shrubby species such as Quercus incana with combined IV of 177.84, followed by Olea ferruginea with IV of 156.87, Otostegia limbata with IV of 142.29, Berberis lyceum with IV 134.4. The other associated species were Cynodon dactylon, Juglans regia, Justicia adhatoda, Sageretia thea, Indigofera heterantha, Apluda mutica, Ailanthus altissima and Morus alba (Fig. 2).

Cluster 2. This group was the richest in terms of species diversity dominated by herbs such as Nepeta cataria with combined IV 44.11, Duchesnea indica 41.96 and Ajuga bracteosa 41.83. The other co-dominant associative species were Stachys parviflora, 
Cirsium vulgare, Heteropogon contortus, Pennisetum orientale, Verbascum, Thapsus and Eragrostis minor (Fig. 2). The vegetation of this group was the indicator of moist temperate zone which could survive during the winter season in the form of seeds bank and had the quick regeneration capability during favorable conditions.

Cluster 3. This group had the association of herbaceous and shrubby vegetation dominated by Rubus fruticosus with combined IV 80.46 followed by Rumex hastatus with IV 50.06 and Andrachne cordifolia with IV 43.77. The other co-dominant associated species were Ziziphus jujuba, Teucrium royleanum, Rubia cordifolia, Ricinus communis, Salvia moorcroftiana, Reinwardtia trigyna, Cannabis sativa and Tagetes minuta (Fig. 2).

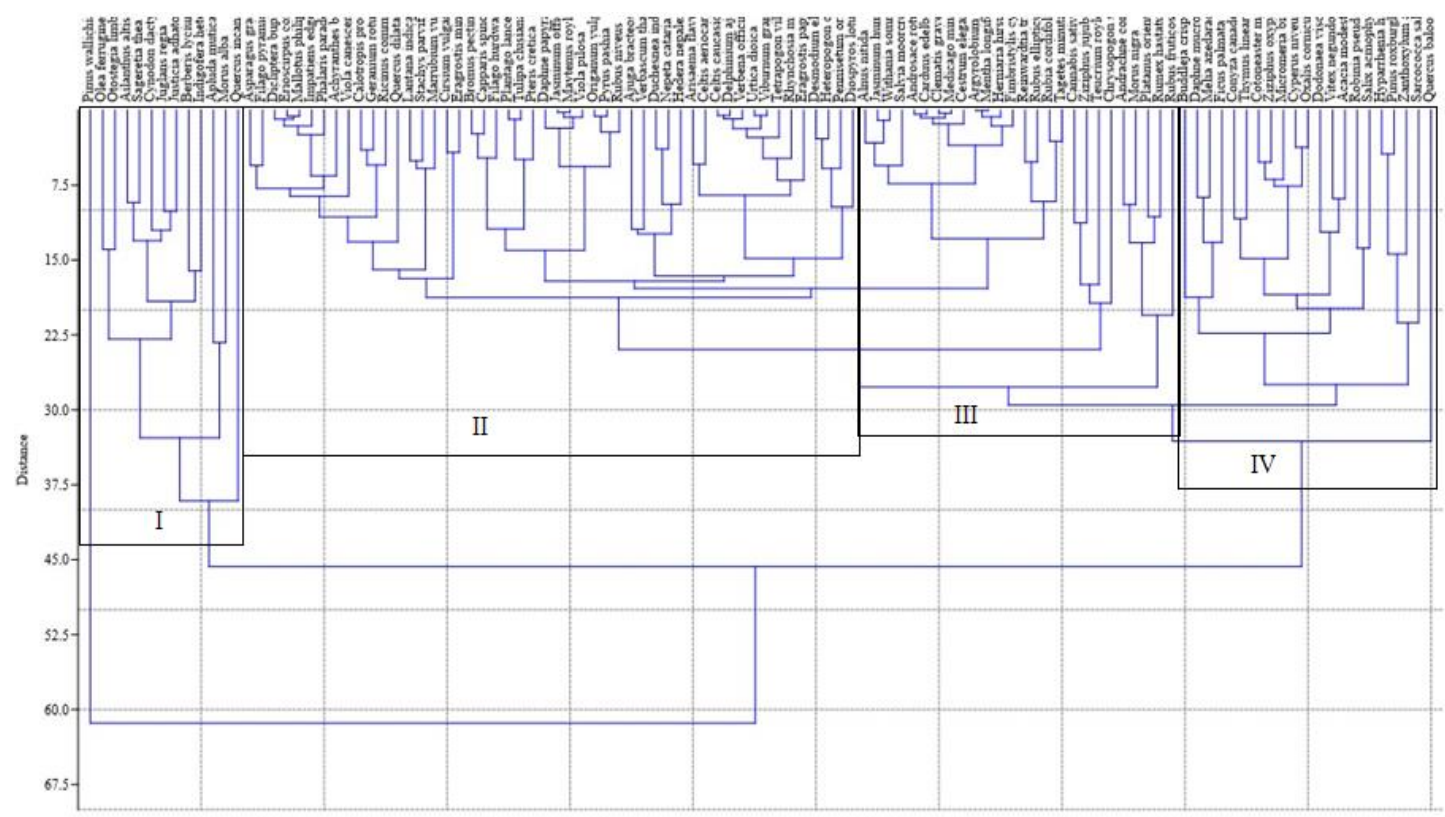

Figure 2. Cluster dendrogram showing the association on the basis of IV values

Cluster 4. This group had mixed type of association led by Sarcococca saligna with combined IV 106.2, Ficus palmata with IV 89.91, Conyza canadensis with IV 86.22 and Oxalis corniculata with IV 71.14. Zanthoxylum armatum, Acacia modesta, Buddleja crispa, Micromeria biflora and Hyparrhenia hirta were the other co-dominant allied species (Fig. 2).

\section{Principal component analysis using PC-Ord}

A PCA biplot was constructed of the plants species under the impact of different sites. In PC-I, species mostly collected from site III, were aggregated in a clumpy manner. In PC-I, Rubus fruticosa is located at a maximum distance from the origin of the biplot while Apluda mutica is located at the same position in PC -IV, both plants species are strongly negative correlated with each other. On the other hand, Querqus dilatata, Salix acmophylla and Viola canescens are negatively correlated to Budleja crispa, Tulipa clusiana and Teucrium stockcianum placed in PC-II. All plants species aggregated in clumps are strongly correlated along the origin (PC-I and PC-II) as shown in Figure 3. 


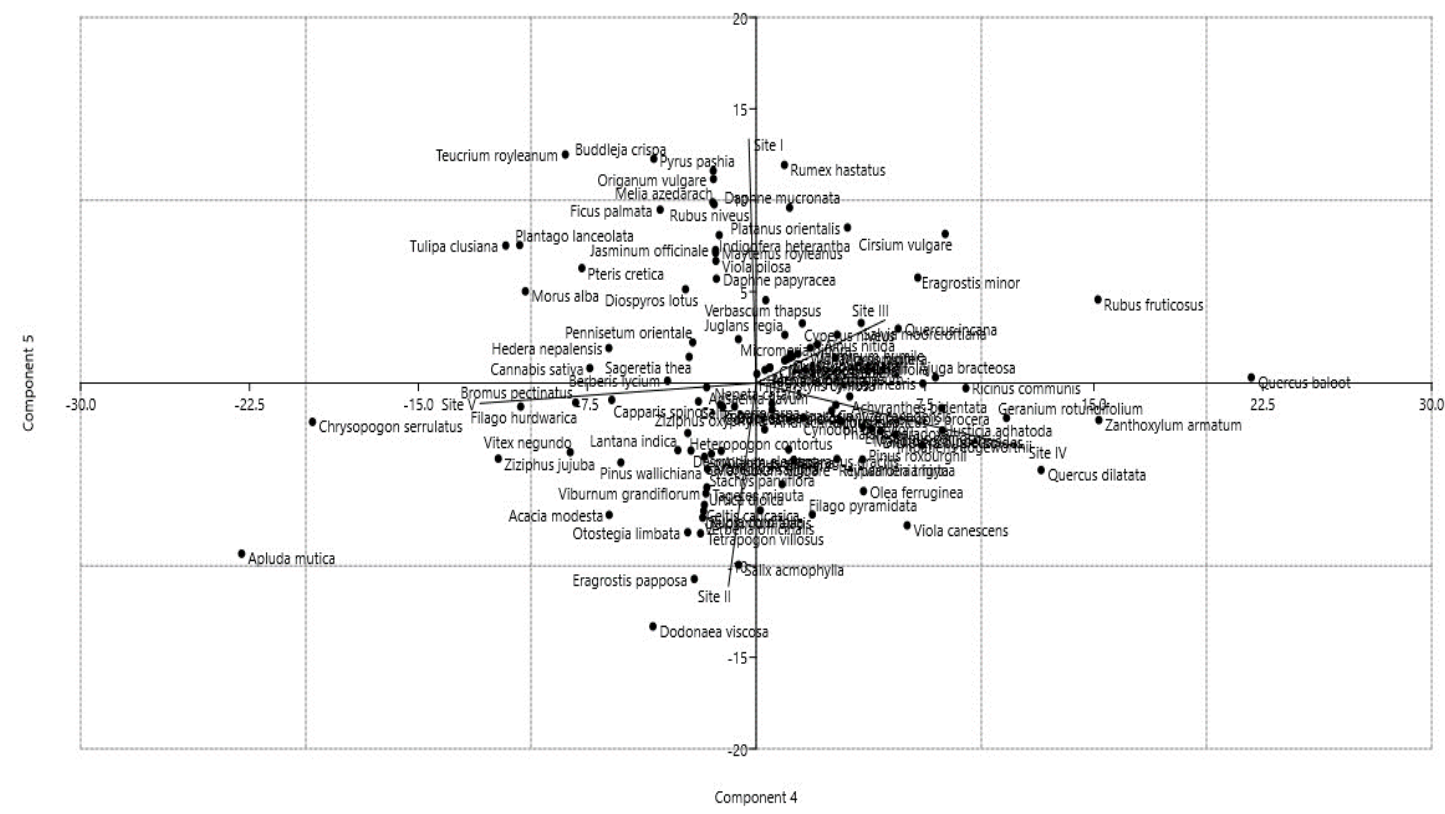

Figure 3. Principal components analysis (PCA) showing the associations based on IV values

\section{Discussion}

The vegetation structure in the present research was classified into fifteen communities of trees, shrubs and herbs based on importance value (IVs), which is in line with the works of Bokhari et al. (2013), Ali et al. (2015), Ilyas et al. (2015), Siddiqui et al. (2015), Ahmad et al. (2016), Khan et al. (2016), Srivastava et al. (2016), Ali et al. (2018), Hussain et al. (2019), Zaman and Badshah (2020). At Site-I, PQJ-BIB-RHC communities were established for trees, shrubs and herbs. Trees communities in all the monitoring sites of area were under sever anthropogenic stress of deforestation, which had created clumping pattern rather than a uniform. The increase in human population and residential construction work in the area were the main reasons that losses the species in the area. Haq et al. (2015), Ahmad et al. (2016), Khan et al. (2016) and Ali et al. (2018) reported similar communities in their respective study areas. Site-II had POQ-BOS-HCH communities. At the monitoring sites, trees and shrubs communities had erratic pattern. The key deriving force that have an effect on the vegetation structure in both sites I and II are soil erosion. The indicator herbaceous species of eroded soil like Achyranthes bidentata, Cannabis sativa, Cyperus niveus, Plantago lanceolata, Rumex hastatus, Salvia moorcroftiana and Verbascum thapsus were grow well. Khan et al. (2010), Akhtar and Bergmeier (2015), Ilyas et al. (2015), Ahmad et al. (2016) and Khan et al. (2016) reported similar communities with different IVs. OQ-ROB-CSC communities dominated site III. This site had a thick and scattered community of Quercus that was mixed with Olea. Quercus community of the area were found disturbed due biotic stress, especially the deforestation. At Site VI, QOJ-SJO-CCT communities were established. Due to deforestation at the North-East aspect, clumpy pitches were found more than the NorthWest with uniform dispersion. The herbaceous community were under the influence of overgrazing that disturbed the species composition. At Site V, QOF-OBS-ACC communities were documented. The edaphic factors are crucial in determining vegetation patterns since each species in a community has a unique response to them. The soil texture 
of the selected sites was silty to clay loam with high content nutrients and had more water that lead to the development of dense vegetation. The nitrogen concentration in site II, IV and V were found high that improves the growth of herbaceous species. The content of phosphorous was found very low in site III but the $\mathrm{pH}$ value is slightly alkaline that may effect on the phosphorus availability to plants. To grow in such condition, the plants may secrete phosphatase enzyme and some organic components to reduce soil $\mathrm{pH}$. This is in line with the findings of Schuur and Matson. (2001), Phillipse, (2003), Wang et al. (2014), Cambrolle et al. (2015) and Zhang et al. (2017). The present study recorded some of endangered, threatened and rear species in the area. The critically endangered species included Caralluma tuberculata, Eremurus himalaicus and Nannorrhops ritchiana while Arisaema flavum, Bergenia ciliata, Myrtus communis, Solanum surattense, Teucrium stocksianum and Vitis jacquemontii were the endangered species. Ajuga bracteosa, Foeniculum vulgare and Jaglans regia were the vulnerable species. These species were ethnomedicinally used by the local community and hakims for treatment of various diseases. Nannorrhops ritchiana which is locally known as 'Mazari' is one of regionally critically endangered species whose leaves were used in making of handicraft such as mats, hand fans, baskets, hats, brooms and trays (Abdullah et al., 2020). Another threatened species is Juglans regia, commonly known as 'Ghoz,' whose wood is widely used in furniture production while its bark is utilized as a tooth brush and in traditional cosmetics. The vegetation structure of tree species was found under the anthropogenic activities of local for the extraction wood. The woody species like Quercus baloot, Quercus incana and Olea ferruginea were found to be decreasing due to its consumption as fuel wood and slow regeneration capacity. The main cause of biodiversity loss in the area is rapid population growth of human which has resulted in the development of roads, buildings and overharvesting of plants.

\section{Conclusion}

The results of vegetation structure in 150 sampling units at 5 monitoring sites are presented in this study. The soil analysis covers 9 parameters with different properties at each location. Cluster analysis PAST and PC-Ord software were used which classified the vegetation into four groups based on quantitative values. Variations in edaphic factors, moisture and temperature causes spatial variation in plant communities at the different monitoring sites. The study area vegetation is threatened, and it must be conserved before it is too late. The impact of anthropogenic activities such as over grazing deforms the herbaceous vegetation structure. Increased agriculture practices also affect the overall forest cover. During the harsh winter season, local residents cut the woody plants which not only decreases the species density in the communities but also speed up the soil erosion. The woody and shrubby plants such as Olea ferruginea, Pinus wallichiana, Pinus roxburghii, Quercus baloot, Quercus incana, Berberis lycium, Buddleja crispa, Daphne mucronata, Indigofera heterantha, Sageretia thea, Viburnum grandiflorum and Ziziphus oxyphylla are under extreme pressure for domestic uses. To combat the effects of deforestation, afforestation projects need to be initiated in the area. The alternative sources for fuel and timber should be provided to protect the vegetation diversity. For regeneration of understory species, a proper grazing management program such as moderate and rotational grazing routine should be ensured. Overpopulation, agricultural practices, developmental work, habitat degradation, deforestation and overgrazing were identified as the main biotic pressures threatening phytodiversity in the study area. As a 
result, it is necessary to protect this phytodiversity for future generations by involving local communities, conservationists, Government and Non-Government Organizations (NGOs). Likely, a comprehensive program must be designed for the measurement of threatened plants diversity and their conservation.

Acknowledgments. This work is a part of the Ph.D. thesis of the first author. The authors acknowledge the assistance of laboratory staff of the Agriculture Research Institute Tarnab Peshawar for physicochemical analysis of soil samples.

Conflict of Interests. The authors declare that they have no competing financial interests.

\section{REFERENCES}

[1] Abdullah, Khan, S. M., Pieroni, A., ul Haq, Z., Ahmad, Z. (2020): Mazri (Nannorrhops ritchiana (Griff) Aitch.): a remarkable source of manufacturing traditional handicrafts, goods and utensils in Pakistan. - J. Ethnobiol. Ethnomed. 16(1): 1-13.

[2] Ahmad, K. S., Ahmad, M., Ahmad, F., Sadia, B. (2016): Edaphic factors as major determinants of plant distribution of temperate Himalayan grasses. - Pak. J. Bot. 48(2): 567-573.

[3] Akhtar, N., Bergmeier, E. (2015): Species richness, alpha and beta diversity of trees, shrubs and herbaceous plants in the woodlands of Swat, Pakistan. - Pak. J. Bot. 47(6): 2107-2113.

[4] Ali, S., Perveen, A., Qaiser, M. (2015): Vegetation structure and edaphology of Mahaban and Malka (District Buner) KPK, Pakistan. - Pak. J. Bot. 4(7): 15-22.

[5] Ali, A., Badshah, L., Hussain, F. (2018): Vegetation structure and threats to montane temperate ecosystems in Hindukush range, Swat, Pakistan. - Appl. Ecol. Env. Res. 16(4): 4789-4811.

[6] Bhat, J. A., Kumar, M., Negi, A. K., Todaria, N. P., Malik, Z. A., Pala, N. A., Shukla, G. (2020): Species diversity of woody vegetation along altitudinal gradient of the Western Himalayas. - Glob. Ecol. Conserv. 24: e01302.

[7] Black, C. A. (1965): Methods of Soil Analysis. - Agron Inc. Madison Wisconsin, USA.

[8] Bokhari, T. Z., Ahmed, M., Siddique, M. F., Khan, Z. (2013): Forest communities of Azad Kashmir, Pakistan. - FUUAST J. Biol. 3(1): 137-145.

[9] Bouyoucos, G. J. (1936): Directions for making mechanical analysis of soils by the Hydrometer Method. - Soil. Sci. 42(3): 225-230.

[10] Brady, N. C. (1990): The Nature and Properties of Soils. 10th ed. - Macmillan Publishing Co. New York.

[11] Braun-Blanquet, J. (1932): Plant sociology. - Transl. Conard, H. S., Fuller, G. D., New York: McGraw-Hill.

[12] Cambrollé, J., Muñoz-Vallés, S., Mancilla-Leytón, J. M., Andrades-Moreno, L., Luque, T., Figueroa, M. E. (2015): Effects of soil physicochemical properties on plant performance of Glaucium flavum Crantz. - Plant and soil 386(1): 185-193.

[13] Cox, G. W. (1967): Laboratory Manual of General Ecology. - W.M.C. Brown Co. Pub. Dubugue Lowa, U.S.A. 165p.

[14] Dengler, J. (2016): Phytosociology. - International Encyclopedia of Geography: People, the Earth, Environment and Technology.

[15] Evangelista, A., Frate, L., Carranza, M. L., Attorre, F., Pelino, G., Stanisci, A. (2016): Changes in composition, ecology and structure of high-mountain vegetation: a re-visitation study over 42 years. - AoB Plants 8 .

[16] Habib, B. A., Raphaël, G., Okkacha, H. (2014): Impact of over-grazing on vegetation and physico-chemical characters of soil steppiques in western Algerian. - Int. J. Basic Appl. Sci. 3(3): 328-334. 
[17] Hailemariam, M. B., Temam, T. D. (2020): Pattern of plant community distribution along the elevational gradient and anthropogenic disturbance in Gole forest, Ethiopia. - Int. J. Ecol., Article ID: 6536374.

[18] Haq, F., Ahmad, H., Iqbal, Z. (2015): Vegetation description and phytoclimatic gradients of subtropical forests of Nandiar Khuwar catchment District Battagram. - Pak. J. Bot. 47(4): 1399-1405.

[19] Hussain, F. (1989): Field \& Laboratory Manual of Plant Ecology. - UGC, Islamabad.

[20] Hussain, W., Badshah, L., Ali, A. (2019): Quantitative aspects of the Koh-e-Safaid range vegetation across the altitudinal gradient in upper Kurram valley, Pakistan. - Appl. Ecol. Env. Res. 17(4): 9905-9924.

[21] Ilyas, M., Qureshi, R., Akhtar, N., Haq, Z. (2015): Vegetation analysis of Kabal Valley, District Swat, Pakistan using multivariate approach. - Pak. J. Bot. 4(7): 77-86.

[22] Khan, N., Ahmad, M., Wahab, M., Nazim, K., Ajaib, M. (2010): Phytosociology, structure and physicochemical analysis of soil in Quercus baloot Griff. Forest District Chitral, Pakistan. - Pak. J. Bot. 42(4): 2429-2441.

[23] Khan, S. M., Harper, D. M., Page, S., Ahmad, H. (2011): Species and community diversity of vascular flora along environmental gradient in Naran Valley: A multivariate approach through indicator species analysis. - Pak. J. Bot. 43(5): 2337-2346.

[24] Khan, A., Ahmad, M., Siddiqui, M. F., Iqbal, J., Wahab, M. (2016): Phytosociological analysis of pine forest at Indus Kohistan, KPK, Pakistan. - Pak. J. Bot. 48(2): 575-580.

[25] Khan, W., Khan, S. M., Ahmad, H., Ahmad, Z., Page, S. (2016): Vegetation mapping and multivariate approach to indicator species of a forest ecosystem: A case study from the Thandiani sub Forests Division (TsFD) in the Western Himalayas. - Ecological Indicators 71: 336-351.

[26] Khan, M., Khan, S. M., Ilyas, M., Alqarawi, A. A., Ahmad, Z., Abd Allah, E. F. (2017): Plant species and community's assessment in interaction with edaphic and topographic factors; an ecological study of the mount Eelum District Swat, Pakistan. - Saudi J. Biol. Sci. 24(4): 778-786.

[27] Kharkwal, G., Mehrotra, P., Rawat, Y. S., Pangtey, Y. P. S. (2005): Phytodiversity and growth form in relation to altitudinal gradient in the central Himalayan (Kumaon) region of India. - Current Science 89(5): 873-878.

[28] Kjeldahl, J. (1883): Neue Methode zur Bestimmung des Stickstoffs in Organischen Körpern. - Z. Anal. Chem. 22: 366-382.

[29] Mandal, G., Joshi, S. P. (2014): Analysis of vegetation dynamics and phytodiversity from three dry deciduous forests of Doon Valley, Western Himalaya, India. - J. Asia Pac. Biodivers. 7(3): 292-304.

[30] Mishra, D., Mishra, T. K., Banerjee, S. K. (1997): Comparative phytosociological and soil physico-chemical aspects between managed and unmanaged lateritic land. - Annals of forestry 5(1): 16-25.

[31] Mota, G. S., Luz, G. R., Mota, N. M., Coutinho, E. S., das Dores Magalhaes Veloso, M., Fernandes, G. W., Nunes, Y. R. F. (2018): Changes in species composition, vegetation structure, and life forms along an altitudinal gradient of rupestrian grasslands in southeastern Brazil. - Flora 238: 32-42.

[32] Motyka, J., Dobrzanski, B., Zawadski, S. (1950): Wstepne badania and lakami polundnlowowschodneij Lubeiszczyzny. - Ann. Univ. M. Curie-Jklodowska 13: 367-447. (Sec. E. 5).

[33] Odum, E. P., Barrett, G. W. (1971): Fundamentals of ecology $3^{\text {rd }}$ ed. - Philadelphia, PA: WB Saunders and Co.

[34] Olsen, S. R., Sommers, L. E. (1982): Phosphorus. - Methods of Soil Analysis, Part 2 (2 ${ }^{\text {nd }}$ ed.) Medison, WI, USA, pp. 406-407.

[35] Oosting, H. J. (1956): The Study of Plant Communities. $-2^{\text {nd }}$ ed., pp. 440. W.H. Freeman and company, San-Francisco, California, USA. 
[36] Phillips, O. L. (2003): Habitat association among Amazonian tree species: a landscape scale approach. - J. Ecol. 91: 757-775.

[37] Pichi-Sermolli, R. E. (1948): An index for establishing the degree of maturity in plant communities. - J. Ecology 36: 85-90.

[38] Rahman, A. U., Khan, S. M., Khan, S., Hussain, A., Rahman, I. U., Iqbal, Z., Ijaz, F. (2016): Ecological assessment of plant communities and associated edaphic and topographic variables in the Peochar valley of the Hindu Kush mountains. - Mountain Research and Development 36(3): 332-341.

[39] Rayan, J. N., Harvey, R. W., Metge, D. W., Larson, J. E. (1997): Transport of bacteriophage PRDI and silica colloids in a sewage contaminated aquifer. - Eos, Transaction of the American Geophysical Union 86, F 231, Presented at the Fall Meeting of the American Geophysical Union.

[40] Schuur, E. A. G., Matson, P. A. (2001): Net primary productivity and nutrient cycling across a mesic to wet precipitation gradient in Hawaiian Montane Forest. - Oecologia 128: 431-442.

[41] Siddiqui, M. F., Salam, A., Ahmad, M., Hussain, M. I., Iqbal, J., Wahab, M. (2015): Present state and future trends of pine forests of Malam Jabba, Swat District, Pakistan. - Pak. J. Bot. 47(6): 2161-2169.

[42] Sorensen, T. (1948): A method of establishing groups of equal amplitudes in plant sociology based on similarity of species content and its application to analyze the vegetation of Darnish commons. - Biol. Skr. 5: 1-34.

[43] Srivastava, R., Mishra, R. M., Awasthi, A. (2016): Phytosociological studies on certain plants of Awarpur (M.S). - Int. J. Pharm. Life. Sci. 7(3): 4930-4936.

[44] Tavili, A., Jafari, M. (2009): Interrelations between plants and environmental variables. Int. J. Environ. Res. 3: 239-246.

[45] Walkley, A. (1947): A critical examination of a rapid method for determining organic carbon in soils: Effect of variation in digestion conditions and of inorganic soil constituents. - Soil. Sci. 63(4): 251-264.

[46] Wang, C., Wang, X. B., Liu, D. W., Wu, H. H., Lü, X. T., Fang, Y. T., Chen, W. X., Luo, W. T., Jiang, P., Shi, J., Yin, H. Q., Zhou, J. Z., Han, X. G., Bai, E. (2014): Aridity threshold in controlling ecosystem nitrogen cycling in arid and semi-arid grasslands. - Nature Communications 5: 4799.

[47] Warger, M. J. A., Morrel, V. E. (1976): Plant species and plant communities: Some conclusion. - In Plant species and plant communities. Proceedings of International Symposium of Nijimegen: 167-175.

[48] Yang, J., El-Kassaby, Y. A., Guan, W. (2020): Multiple ecological drivers determining vegetation attributes across scales in a mountainous dry valley, Southwest China. - Forests 11(11): 1140.

[49] Zaman, A., Badshah, L. (2020): Vegetation dynamics along an elevational gradient in Terich valley, Chitral Hindu Kush range, northern Pakistan. - Appl. Ecol. Env. Res. 18(5): 6099-6119.

[50] Zerwes, C. M., Rempel, C., Schneider, J. K., Maranho, L. T. (2018): Importance of the review on floristic and phytosociological studies of the arboreal stratum of the seasonal deciduous forest of the Serra Geral slope, Rio Grande do Sul, Brazil, to support proposals for sustainable management. - Ciência e Natura 40: 41.

[51] Zhang, X. N., Yang, X. D., Li, Y., He, X. M., Lv, G. H., Yang, J. J. (2017): Influence of edaphic factors on plant distribution and diversity in the arid area of Xinjiang, Northwest China. - Arid Land Res. Manag. 32(1): 38-56. 